\title{
SERIES INEQUALITIES INVOLVING CONVEX FUNCTIONS
}

\author{
BY
}

\section{CHRISTOPHER O. IMORU( $\left.{ }^{1}\right)$}

ABSTRACT. The object of this paper is to extend some recent generalizations of Petrovic's inequality by Vasić and others. We shall also use our technique to obtain some results which have interesting applications in the theory of Fourier series as well as the theory of approximations.

1. Introduction. M. Petrovic' [9] (see also Mitrinovic' [7]) has obtained the following result:

If $\phi$ is a convex function on the segment $\bar{I}=[0, a]$, if $x_{i} \in \bar{I}, i=$ $1, \cdots, n$ and $x_{1}+\cdots+x_{n} \in \bar{I}$, then

$$
\phi\left(x_{1}\right)+\cdots+\phi\left(x_{n}\right) \leqslant \phi\left(x_{1}+\cdots+x_{n}\right)+(1-n) \phi(0)
$$

with equality if and only if either $n=1$ or $x_{1}=\cdots=x_{n}=0$.

Recently, there have been various generalizations of this inequality, some of which were given by Marković [6], Vasić [11], and Keđkić and Lacković [5]. (For further references on these and other generalizations, see Mitrinović [7].)

The purpose of this paper is to give an improved version of this elegant inequality of M. Petrović and to discuss in detail some of the consequences and applications of our main results.

2. Generalizations of Vasic's inequality. Suppose $\left(a_{k}\right)_{1 \leqslant k \leqslant n} \subseteq[1, \infty]$. Then the inequality

$$
\sum_{k=1}^{n} a_{k} \phi\left(x_{k}\right) \leqslant \phi\left(\sum_{k=1}^{n} a_{k} x_{k}\right)-\left(1-\sum_{k=1}^{n} a_{k}\right) \phi(0)
$$

holds for every continuous convex function $\phi$ defined on $\bar{I}=[0, \infty]$ whenever $x_{k} \in \vec{I}, k=1, \cdots, n$ and $\sum_{k=1}^{n} a_{k} x_{k} \in I=(0, \infty)$.

Received by the editors October 27, 1973 and, in revised form, December 21, 1973. AMS (MOS) subject classifications (1970). Primary 26A86.

Key words and phrases. Jensen-Steffensen inequality, continuous convex functions, monotonic functions.

(1) The results of this paper are part of the author's Ph. D. dessertation written under Professor R. P. Boas, Jr. at Northwestern University, Evanston, Illinois in 1972. The author wishes to thank Professor Boas for his advice and encouragement. 
This inequality was proved by P. M. Vasic [11], who made use of the property of convex functions. He obtained from this inequality generalizations of certain inequalities of D. Markovic [6] and S. Gatti [2]. Further generalizations of his results were also given by Keckić and Lacković [5].

We shall obtain in this section some generalizations of Vasic's results. Indeed, our results will show that Vasic's results and other existing similar results hold under weaker conditions. Throughout this paper, $a=\left(a_{k}\right)_{-m \leqslant k \leqslant n}$ will denote a sequence of real numbers so that $\sum_{k=1}^{n} a_{k} \geqslant 1$, with equality if and only if $n=1$. In order to prove our main results, we shall make essential use of the following inequality of Steffensen [10] (see also [7] and [8]), which we state, without proof, as a lemma.

LEMMA (THE JENSEN-STEFFENSEN INEQUALITY FOR SUMS). If $\phi$ is $a$ continuous convex function on $\bar{I}=[a, b],-\infty \leqslant a<b \leqslant \infty$, if $\left(z_{k}\right)_{-m \leqslant k \leqslant n} \subseteq \bar{I}$ never decreases and $\left(c_{k}\right)_{-m \leqslant k \leqslant n}$ satisfies the inequality

$$
0 \leqslant \sum_{k=\nu}^{n} c_{k} \leqslant \sum_{k=-m}^{n} c_{k} \quad(-m \leqslant \nu \leqslant n)
$$

with $\Sigma_{k=-m}^{n} c_{k}>0$, then

$$
\phi\left(\frac{\sum_{k=-m}^{n} c_{k} z_{k}}{\sum_{k=-m}^{n} c_{k}}\right) \leqslant \frac{\Sigma_{k=-m}^{n} c_{k} \phi\left(z_{k}\right)}{\sum_{k=-m}^{n} c_{k}} .
$$

REMARK. For the necessary and sufficient conditions for inequality (2.3) to be strict, see [3].

Since the conditions for strict inequality in the results of this paper follow from those of the lemma, we shall always be tacit about the necessary and sufficient conditions for equality.

THEOREM 2.1. Let $\left(x_{k}\right)_{-n \leqslant k \leqslant n}$ be a nondecreasing sequence of points in $(a, b)$ where $-\infty \leqslant a<0<b \leqslant \infty$ and $0 \leqslant x_{k}<b$ for $k \geqslant 1$. If

$$
\sum_{k=1}^{n} a_{k} x_{k} \in[0, b) \text { and } x_{k}\left[1-x_{0}\left(\sum_{k=1}^{n} a_{k} x_{k}\right)^{-1}\right]+x_{-k} \in(a, b)
$$

where $a_{k}>0,1 \leqslant k \leqslant n$, then the inequality

$$
\begin{array}{r}
\sum_{k=1}^{n} a_{k} \phi\left[x_{k}-x_{k} x_{0}\left(\sum_{k=1}^{n} a_{k} x_{k}\right)^{-1}+x_{-k}\right] \\
\leqslant \phi\left(\sum_{k=1}^{n} a_{k} x_{k}\right)-\phi\left(x_{0}\right)+\sum_{k=1}^{n} a_{k} \phi\left(x_{-k}\right)
\end{array}
$$


holds for every continuous convex function $\phi$ defined on $(a, b)$. The inequality is strict unless $x_{-n}=\cdots=x_{n}=0$.

Proof. Consider the particular case $m=n=1$ of the lemma. Set

$$
\begin{gathered}
z_{-1}=x_{-k}, \quad 1 \leqslant k \leqslant n, \quad z_{0}=x_{0}, \quad z_{1}=\sum_{k=1}^{n} a_{k} x_{k}, \\
c_{-1}=a_{k}, \quad 1 \leqslant k \leqslant n, \quad c_{0}=-\left(\sum_{k=1}^{n} a_{k} x_{k}\right)^{-1} a_{k} x_{k}, \quad c_{1}=\left(\sum_{k=1}^{n} a_{k} x_{k}\right)^{-1} a_{k} x_{k} .
\end{gathered}
$$

It is readily verified that the conditions for the validity of inequality (2.3) are fulfilled. So, we have for $1 \leqslant k \leqslant n$,

$$
\begin{aligned}
& a_{k} \phi\left[x_{-k}-x_{k} x_{0}\left(\sum_{k=1}^{n} a_{k} x_{k}\right)^{-1}+x_{k}\right] \\
& \quad \leqslant a_{k} \phi\left(x_{-k}\right)-a_{k} x_{k}\left(\sum_{k=1}^{n} a_{k} x_{k}\right)^{-1} \phi\left(x_{0}\right)+a_{k} x_{k}\left(\sum_{k=1}^{n} a_{k} x_{k}\right)^{-1} \phi\left(\sum_{k=1}^{n} a_{k} x_{k}\right) .
\end{aligned}
$$

Summing up this series of inequalities over $k, 1 \leqslant k \leqslant n$, we obtain the assertion of the theorem.

By the same method, the following theorem can be proved.

THEOREM 2.2. Let $\left(x_{k}\right)_{-n \leqslant k \leqslant n}$ be a nondecreasing sequence of points in $(b, a)$ where $-\infty \leqslant b<0<a \leqslant \infty$ and $b<x_{-k} \leqslant 0$ for $k \geqslant 1$. If

$$
\sum_{k=1}^{n} a_{-k} x_{-k} \in(b, 0] \quad \text { and } x_{-k}\left[1-x_{0}\left(\sum_{k=1}^{n} a_{-k} x_{-k}\right)^{-1}\right]+x_{k} \in(b, a)
$$

where $a_{-k}>0,1 \leqslant k \leqslant n$, then the inequality

$$
\begin{array}{r}
\sum_{k=1}^{n} a_{-k} \phi\left[x_{-k}\left\{1-x_{0}\left(\sum_{k=1}^{n} a_{-k} x_{-k}\right)^{-1}\right\}+x_{k}\right] \\
\leqslant \phi\left(\sum_{k=1}^{n} a_{-k} x_{-k}\right)-\phi\left(x_{0}\right)+\sum_{k=1}^{n} a_{-k} \phi\left(x_{k}\right)
\end{array}
$$

holds for every continuous convex function $\phi$ defined on $(b, a)$. The inequality is strict unless $x_{-n}=\cdots=x_{n}=0$.

Suppose $x_{-k}=x_{0}=0,1 \leqslant k \leqslant n$, and $a_{k} \geqslant 1,1 \leqslant k \leqslant n$, then inequality (2.4) reduces to inequality (2.1). Of course, under the weaker assumptions $a_{k}>0,1 \leqslant k \leqslant n$, with $\Sigma_{k=1}^{n} a_{k} \geqslant 1$, one obtains the improved version of inequality (2.1), namely: 


$$
\sum_{k=1}^{n} a_{k} \phi\left(x_{k}\right) \leqslant \phi\left(\sum_{k=1}^{n} a_{k} x_{k}\right)-\left(1-\sum_{k=1}^{n} a_{k}\right) \phi(0) .
$$

A similar inequality analogous to inequality (2.6) can be obtained from Theorem 2.2. Indeed, if we put $x_{k}=x_{0}=0,1 \leqslant k \leqslant n$, in inequality (2.5), we obtain

$$
\sum_{k=1}^{n} a_{-k} \phi\left(x_{-k}\right) \leqslant \phi\left(\sum_{k=1}^{n} a_{-k} x_{-k}\right)-\left(1-\sum_{k=1}^{n} a_{-k}\right) \phi(0),
$$

where $\left(x_{-k}\right)_{1 \leqslant k \leqslant n}$ is a nonpositive decreasing sequence in $(b, 0]$ and $a_{-k}>0, \quad 1 \leqslant k \leqslant n, \quad \Sigma_{k=1}^{n} a_{-k} \geqslant 1$.

The proof of our next result is based on the following:

THEOREM 2.3. Let $\left(\alpha_{k}\right)_{-m \leqslant k \leqslant n} \subseteq R_{+}, \alpha_{n}>0$, and suppose $\left(\xi_{k}\right)_{-m \leqslant k \leqslant n}$ is a sequence of increasing real numbers in $I=(a, b)$ where $-\infty \leqslant a<b \leqslant \infty$. If $\Sigma_{k=-m+1}^{n} \alpha_{k} \geqslant 1$, with equality if and only if $n=1, \alpha_{n}+\Sigma_{k=-m}^{\nu} \alpha_{k} \geqslant 0$ $(-m \leqslant \nu \leqslant n-1)$ and $\xi_{n-1} \leqslant\left(\sum_{k=-m}^{n} \alpha_{k}\right)^{-1} \sum_{k=-m}^{n} \alpha_{k} \xi_{k},\left(\sum_{k=-m}^{n} \alpha_{k}\right)^{-1} \sum_{k=-m}^{n} \alpha_{k} \xi_{k}$ $\in I$, then the inequality

$$
\sum_{k=-m}^{n} \alpha_{k} \phi\left(\xi_{k}\right) \leqslant\left(\sum_{k=-m}^{n} \alpha_{k}\right) \phi\left(\frac{\Sigma_{k=-m}^{n} \alpha_{k} \xi_{k}}{\sum_{k=-m}^{n} \alpha_{k}}\right)
$$

holds for every continuous convex function $\phi$ defined on $I$. The inequality is strict unless $\xi_{k}=0,-m \leqslant k \leqslant n$.

Proof. Put $c_{k}=-\alpha_{k},-m \leqslant k \leqslant n-1, c_{n}=\Sigma_{k=-m}^{n} \alpha_{k}, z_{k}=\xi_{k}$, $-m \leqslant k \leqslant n-1$ and $z_{n}=\left(\sum_{k=-m}^{n} \alpha_{k}\right) \sum_{k=-m}^{n} \alpha_{k} \xi_{k}$ in the Jensen-Steffensen inequality for sums (i.e. the lemma). Then the conditions for the validity of inequality (2.3) are fulfilled. Hence, the assertion of the theorem is immediate.

Suppose $m=0, \alpha_{0}=1-\Sigma_{k=1}^{n} \alpha_{k}$ and $\xi_{0}=0$. Then, we obtain the following special case of the theorem.

COROLlaRY 2.4. Let $\left(a_{k}\right)_{1 \leqslant k \leqslant n} \subseteq R_{+}$, and suppose the sequence $\left(x_{k}\right)_{1 \leqslant k \leqslant n} \subseteq I=(a, b),-\infty \leqslant a<b \leqslant \infty$, never decreases. If $\sum_{k=1}^{n} a_{k} x_{k} \in I$, then the inequality

$$
\sum_{k=1}^{n} a_{k} \phi\left(x_{k}\right) \leqslant \phi\left(\sum_{k=1}^{n} a_{k} x_{k}\right)-\left(1-\sum_{k=1}^{n} a_{k}\right) \phi(0)
$$

holds for every continuous convex function $\phi$ defined on $I$. The inequality is strict unless $x_{k}=0,1 \leqslant k \leqslant n$.

This corollary suggests that the consequences of Theorems 2.1 and 2.2, when combined, yield: 
Proposition 2.5. Let $\mathrm{a}=\left(a_{-m}, \cdots, a_{n}\right)$ and $\mathrm{x}=\left(x_{-m}, \cdots, x_{n}\right)$ be arbitrary sequences of numbers which satisfy:

$$
\begin{aligned}
& a_{0}>-1, \quad a_{k} \geqslant 0, \quad k \neq 0, \quad \sum_{k=1}^{m} a_{-k}>1, \quad \sum_{k=1}^{n} a_{k}>1, \\
& \left(x_{-k}\right)_{0 \leqslant k \leqslant m} \subseteq I^{\prime}=\left(b^{\prime}, 0\right], \quad\left(x_{k}\right)_{0 \leqslant k \leqslant n} \subseteq I=[0, b),
\end{aligned}
$$

$$
\sum_{k=1}^{n} a_{k} x_{k} \in I, \quad \sum_{k=1}^{m} a_{-k} x_{-k} \in I^{\prime}
$$

and

$$
\left(x_{k}\right)_{-m \leqslant k \leqslant n} \text { never decreases. }
$$

If $\phi$ is a continuous convex function on $I^{\prime} \cup I$, then the inequality

$$
\sum_{k=-m}^{n} a_{k} \phi\left(x_{k}\right) \leqslant \phi\left(\sum_{k=-m}^{n} a_{k} x_{k}\right)-\left(1-\sum_{k=-m}^{n} a_{k}\right) \phi(0)
$$

holds with equality if and only if $x_{-m}=\cdots=x_{n}=0$.

Proof. From Theorems 2.1 and 2.2, we have respectively:

$$
\sum_{k=1}^{n} a_{k} \phi\left(x_{k}\right) \leqslant\left(\sum_{k=1}^{n} a_{k} x_{k}\right)-\left(1-\sum_{k=1}^{n} a_{k}\right) \phi(0)
$$

$$
\sum_{k=1}^{m} a_{-k} \phi\left(x_{-k}\right) \leqslant\left(\sum_{k=1}^{m} a_{-k} x_{-k}\right)-\left(1-\sum_{k=1}^{m} a_{-k}\right) \phi(0) .
$$

Adding $a_{0} \phi\left(x_{0}\right)$ to the sum of these inequalities, we get

$$
\begin{aligned}
\sum_{k=-m}^{n} a_{k} \phi\left(x_{k}\right) \leqslant & \phi\left(\sum_{k=1}^{m} a_{-k} x_{-k}\right)+a_{0} \phi\left(x_{0}\right)+\phi\left(\sum_{k=1}^{n} a_{k} x_{k}\right) \\
& -\left(2-\sum_{k=1}^{m} a_{-k}-\sum_{k=1}^{n} a_{k}\right) \phi(0) .
\end{aligned}
$$

Taking $m=1, n=1$ and $\alpha_{-1}=\alpha_{1}=1, \alpha_{0}=a_{0}, \xi_{-1}=\Sigma_{k=1}^{m} a_{-k} x_{-k}, \xi_{0}=$ $x_{0}$ and $\xi_{1}=\sum_{k=1}^{n} a_{k} x_{k}$ in Theorem 2.3, we get from inequality (2.8), $n=1$

$$
\phi\left(\sum_{k=1}^{m} a_{-k} x_{-k}\right)+a_{0} \phi\left(x_{0}\right)+\phi\left(\sum_{k=1}^{n} a_{k} x_{k}\right) \leqslant \phi\left(\sum_{k=-m}^{n} a_{k} x_{k}\right)+\left(1+a_{0}\right) \phi(0) .
$$

Using this inequality in the above inequality, we obtain inequality (2.9).

From inequality (2.9) for $m=0, x_{0}=0$ and $a_{k}=\lambda^{-1} p_{k} \geqslant 0\left(\lambda>0, p_{k} \geqslant 0\right)$, $1 \leqslant k \leqslant n$, we get the inequality 


$$
\sum_{k=1}^{n} p_{k} \phi\left(x_{k}\right) \leqslant \lambda \phi\left(\frac{1}{\lambda} \sum_{k=1}^{n} p_{k} x_{k}\right)-\left(\lambda-\sum_{k=1}^{n} p_{k}\right) \phi(0) \text {. }
$$

This inequality was obtained by Vasić (see $[8,3.9 .58])$ when $\lambda$ is a positive integer and

$$
\begin{gathered}
\sum_{k=1}^{n_{1}-1} p_{k} x_{k}=\sum_{k=n_{1}}^{n_{2}-1} p_{k} x_{k}=\cdots=\sum_{k=n}^{n} p_{k} x_{k} ; \quad p_{k} \geqslant 1,1 \leqslant k \leqslant n, \\
n_{0}=1, \quad n_{\lambda}-1=n .
\end{gathered}
$$

This result is easily obtained by a repeated application of inequality (2.6).

Our next result shows that the conditions $p_{k} \geqslant 1,1 \leqslant k \leqslant n$, and $\lambda$ is an arbitrary positive integer can be relaxed.

THEOREM 2.6. Suppose $\left(\alpha_{k}\right)_{0 \leqslant k \leqslant r} \subseteq(-\infty, \infty)$ is an arbitrary sequence of real numbers which satisfies $\alpha_{0}=0$, and $1=n_{\alpha_{0}} \leqslant n_{\alpha_{1}}-1 \leqslant \cdots \leqslant n_{\alpha_{r}}-1=n$, $\left(n_{\alpha_{k}}\right)_{0 \leqslant k \leqslant r}$ are positive integers.

Let $\phi$ be a continuous convex function on $I=[0, b)$ and let $\mathbf{x}=$ $\left(x_{1}, \cdots, x_{n}\right)$ and $\mathrm{p}=\left(p_{1}, \cdots, p_{n}\right)$ be two arbitrary sequences of real numbers, $p_{k} \geqslant 0,1 \leqslant k \leqslant n$, with $\Sigma_{k=1}^{n} p_{k} \geqslant\left|\alpha_{r}\right|$. If

$$
\sum_{k=1}^{n_{\alpha_{1}}{ }^{-1}} p_{k} x_{k}=\sum_{k=n_{\alpha_{1}}}^{n_{\alpha_{2}}{ }^{-1}} p_{k} x_{k}=\cdots=\sum_{k=n_{\alpha_{r}-1}}^{n} p_{k} x_{k} .
$$

Then the inequality

$$
\sum_{k=1}^{n} p_{k} \phi\left(x_{k}\right) \leqslant\left|\alpha_{r}\right| \phi\left(\frac{1}{\left|\alpha_{r}\right|} \sum_{k=1}^{n} p_{k} x_{k}\right)-\left(\left|\alpha_{r}\right|-\sum_{k=1}^{n} p_{k}\right) \phi(0)
$$

holds whenever $\left(x_{k}\right)_{1 \leqslant k \leqslant n} \subseteq I=[0, b)$ is nondecreasing and $\left|\alpha_{r}\right|^{-1} \sum_{k=1}^{n} p_{k} x_{k} \in I$.

It is readily verified that this theorem generalizes inequality (2.12). The case $\left|\alpha_{r}\right|=\lambda=1$ is the improved version of inequality (2.6). Since this theorem can be obtained by a repeated application of inequality (2.6), the proof is, therefore, omitted.

Observe however than an extension of inequality (2.11) can be obtained as a direct consequence of Theorem 2.3. Under more restrictive hypotheses on the $p_{k}$ 's and $x_{k}$ 's, the conclusion of Theorem 2.6 holds when condition $\left(2.12^{\prime}\right)$ is relaxed.

We state this result as follows:

THEOREM 2.7. Let $\phi$ be a continuous convex function of $I=(a, b)$, 
$-\infty \leqslant a<b \leqslant \infty$, and suppose $\left(a_{k}\right)_{0<k<n} \subseteq R, a_{n}+\Sigma_{k=0}^{\nu} a_{k} \geqslant 0$ $(0 \leqslant \nu \leqslant n-1)$ and $\Sigma_{k=0}^{n} a_{k} \geqslant 1$, with equality if and only if $n=1$. If $\left(x_{k}\right)_{0 \leqslant k<n}$ is an arbitrary sequence of real numbers in $I$ satisfying

$$
x_{n-1} \leqslant\left(\sum_{k=0}^{n} a_{k}\right)^{-1} \sum_{k=0}^{n} a_{k} x_{k}, \quad \text { where }\left(\sum_{k=0}^{n} a_{k}\right)^{-1} \sum_{k=0}^{n} a_{k} x_{k} \in I,
$$

then the inequality

$$
\sum_{k=1}^{n} a_{k} \phi\left(x_{k}\right) \leqslant \lambda \phi\left(\frac{1}{\lambda} \sum_{k=1}^{n} a_{k} x_{k}\right)-\left(\lambda-\sum_{k=1}^{n} a_{k}\right) \phi(0)
$$

is valid, where $\phi$ is an arbitrary number which satisfies $a_{0}=\lambda-\sum_{k=1}^{n} a_{k} \leqslant 0$, $\lambda \in R_{+}$.

Proof. Put $m=0, \alpha_{k}=a_{k}, 0 \leqslant k \leqslant n, \xi_{0}=0$ and $\xi_{k}=x_{k}, 1 \leqslant k$ $\leqslant n$, in Theorem 2.3. The assertion of the theorem is, therefore, immediate.

Inequalities (2.11) and (2.11') are further generalizations of Vasic's generalizations as well as Kečvić and Lacković's generalizations of an inequality of S. Gatti and certain inequalities of Marković, Marshall-Olkin-Proscham. These inequalities can be obtained from inequalities (2.11) and (2.11') for the appropriate choices of $\lambda, p_{k}$ and $a_{k}(1 \leqslant k \leqslant n)$. Our method of proofs, which differs sharply from the existing ones, clearly shows that the inequalities of Vasic' and others are derivable from the Jensen-Steffensen inequality. Our next result combines the Jensen inequality and the extension of Petrović's inequality as given by Proposition 2.5. Of course, this result, like our other results, is not only an extension but an improvement of a recent result proved by Janić and Vasić [4].

THEOREM 2.8. Let the derivative $\phi^{\prime}$ of the function $\phi$ defined on $I=$ $[0,2 b]$ be continuous convex on $[0,2 b]$. If $\left(x_{k}\right)_{1 \leqslant k \leqslant n} \subseteq[0, b], p_{k} \geqslant 0$, $1 \leqslant k \leqslant n, \quad \sum_{k=1}^{n} p_{k} \geqslant 1$ with equality if and only if $n=1$, and $\sum_{k=1}^{n} p_{k} x_{k} \in$ $[0, b]$, then we have

$$
\begin{aligned}
& \sum_{k=1}^{n} p_{k} {\left[\phi\left(\frac{\sum_{k=1}^{n} p_{k} x_{k}}{\sum_{k=1}^{n} p_{k}}\right)-\phi\left(2 b-\frac{\sum_{k=1}^{n} p_{k} x_{k}}{\sum_{k=1}^{n} p_{k}}\right)\right] } \\
& \geqslant \sum_{k=1}^{n} p_{k} \phi\left(x_{k}\right)-\sum_{k=1}^{n} p_{k} \phi\left(2 b-x_{k}\right) \\
& \geqslant \phi\left(\sum_{k=1}^{n} p_{k} x_{k}\right)-\phi\left(2 b-\sum_{k=1}^{n} p_{k} x_{k}\right) \\
&+\left(1-\sum_{k=1}^{n} p_{k}\right)[\phi(2 b)-\phi(0)] .
\end{aligned}
$$


There is equality in the first inequality if and only if $x_{k}=$ $\left(\sum_{k=1}^{n} p_{k}\right)^{-1} \sum_{k=1}^{n} p_{k} x_{k}, 1 \leqslant k \leqslant n$, while in the second inequality if and only if $x_{k}=0,1 \leqslant k \leqslant n$.

PROOF. Since $\phi^{\prime \prime \prime}(\xi) \geqslant 0, \xi \in[0,2 b]$, we have, by the first mean value theorem, $F: u \rightarrow \phi(2 b-u)-\phi(u)$ is a continuous convex function in $[0, b]$. Hence, an application of Jensen's inequality for sums and that of the improved inequality of Petrovic (see inequality (2.6)), gives

$$
F\left(\frac{\sum_{k=1}^{n} p_{k} x_{k}}{\sum_{k=1}^{n} p_{k}}\right) \leqslant \frac{\sum_{k=1}^{n} p_{k} F\left(x_{k}\right)}{\sum_{k=1}^{n} p_{k}} \leqslant \frac{F\left(\sum_{k=1}^{n} p_{k} x_{k}\right)-\left(1-\sum_{k=1}^{n} p_{k}\right) F(0)}{\sum_{k=1}^{n} p_{k}} .
$$

The proof of the theorem is, therefore, obvious.

In a similar way, using Theorem 2.7 , we obtain the inequality:

$$
\begin{aligned}
\sum_{k=1}^{n} p_{k}[\phi & \left.\left(\frac{\sum_{k=1}^{n} p_{k} x_{k}}{\sum_{k=1}^{n} p_{k}}\right)-\phi\left(2 b-\frac{\sum_{k=1}^{n} p_{k} x_{k}}{\sum_{k=1}^{n} p_{k}}\right)\right] \\
\geqslant & \sum_{k=1}^{n} p_{k} \phi\left(x_{k}\right)-\sum_{k=1}^{n} p_{k} \phi\left(2 b-x_{k}\right) \\
\geqslant & \lambda \phi\left(\frac{1}{\lambda} \sum_{k=1}^{n} p_{k} x_{k}\right)-\lambda \phi\left(2 b-\frac{1}{\lambda} \sum_{k=1}^{n} p_{k} x_{k}\right) \\
& +\left(\lambda-\sum_{k=1}^{n} p_{k}\right)[\phi(2 b)-\phi(0)]
\end{aligned}
$$

where

$$
0 \leqslant \sum_{k=\nu}^{n} p_{k} \leqslant \sum_{k=1}^{n} p_{k}, \quad 1 \leqslant \nu \leqslant n, \text { with } \sum_{k=1}^{n} p_{k} \geqslant \lambda, \lambda \geqslant 1,
$$

and

$$
x_{n-1} \leqslant\left(\sum_{k=1}^{n} p_{k}\right)^{-1} \sum_{k=1}^{n} p_{k} x_{k} \quad \text { where } p_{0}=\lambda-\sum_{k=1}^{n} p_{k}<0 .
$$

3. On a theorem of Boas. As an application of our results, we shall obtain the following result which has many interesting applications in the theory of approximation as well as Fourier series.

THEOREM 3.1. Let $K(x, y)$ be nonnegative homogeneous of degree - 1 . Let $\int_{-\infty}^{\infty} K(1, u) d u<\infty$ and suppose that either

(i) $K(m, n)$ decreases in both arguments, $K(0, n)=0$ for each $n$, or 
(ii) $K(m, n)=0$ for $m>n, K(0, n) \geqslant 1$ and $K(m, n)$ decreases in both arguments for $m \leqslant n$, or

(iii) $K(0, n)=0$ for each $n, K(m, n)=0$ for $m<n$ and $K(m, n)$ decreases in $m$ and is monotonic in $n$ for $m \geqslant n$.

Then, for any arbitrary positive number $\lambda$ such that $\Sigma_{m=-r}^{s} K(m, n) \lambda^{-1}>1$, $-\infty \leqslant r, s \leqslant \infty$, we have

$$
\begin{gathered}
\sum_{n=-\infty}^{\infty}\left[\sum_{m=-\infty}^{\infty} K(m, n) a_{m}\right]^{p} \geqslant A(p, \lambda, K) \sum_{m=-\infty}^{\infty} a_{m}^{p}, \quad p>1 ; \\
\sum_{n=-\infty}^{\infty}\left[\sum_{m=-\infty}^{\infty} K(m, n) a_{m}\right]^{p} \leqslant A(p, \lambda, K) \sum_{m=-\infty}^{\infty} a_{m}^{p}, \quad 0<p<1,
\end{gathered}
$$

provided $\left(a_{m}\right)_{-\infty \leqslant m \leqslant \infty} \subseteq R$ is monotonic nondecreasing, $\left(a_{m}\right)_{0 \leqslant m \leqslant \infty} \subseteq R_{+}$ and $\left(a_{m}\right)_{-\infty \leqslant m<0} \subseteq R_{-}$. The constants $A(p, \lambda, K)=\int_{R-[0,1)} K(1, u) d u$ in case (i), $A(p, \lambda, K)=\int_{1}^{\infty} K(1, u) d u$ in case (ii) and $A(p, \lambda, K)=\int_{-\infty}^{1} K(1, u) d u$ in case (iii) are not necessarily the best possible.

Boas' theorem [1, Theorem 2] follows from Theorem 3.1 when $\left(a_{m}\right)_{m \leqslant 0}$ is a null sequence (i.e. $\left.a_{m}=0,-\infty \leqslant m \leqslant 0\right)$ and the sequence $\left(a_{m}\right)_{m>0}$ is quasimonotonic decreasing in $R_{+}$(i.e. $\left(a_{m} m^{-\lambda}\right)_{m \geqslant 0}$ is monotonic decreasing for some nonnegative number $\lambda$ ). A somewhat stronger version of this theorem can be obtained by using Theorem 2.3.

Proof of THEOREM 3.1. Let $\phi(u)=u^{p} \quad(p>1$ or $p<0)$ and $x_{m}=$ $a_{m}$ and $\alpha_{m}=K(m, n) \lambda^{-1}$ in the inequality

$$
\sum_{m=-r}^{s} \alpha_{m} \phi\left(x_{m}\right) \leqslant \phi\left(\sum_{m=-r}^{s} \alpha_{m} x_{m}\right)-\left(1-\sum_{m=-r}^{s} \alpha_{m}\right) \phi(0)
$$

(see inequality (2.9)). We have

$$
\left[\sum_{m=-r}^{s} K(m, n) a_{m}\right]^{p} \geqslant \lambda^{p-1} \sum_{m=-r}^{s} K(m, n) a_{m}^{p} .
$$

Letting $r$ and $s$ approach infinity, we get on summing the result over $n$, $-\infty \leqslant n \leqslant \infty$,

$$
\begin{aligned}
\sum_{n=-\infty}^{\infty}\left[\sum_{m=-\infty}^{\infty} K(m, n) a_{m}\right]^{p} & \geqslant \lambda^{p-1} \sum_{n=-\infty}^{\infty} \sum_{m=-\infty}^{\infty} K(m, n) a_{m}^{p} \\
& =\lambda^{p-1} \sum_{m=-\infty}^{\infty} a_{m}^{p} \sum_{n=-\infty}^{\infty} K(m, n) .
\end{aligned}
$$

In case (i), we have for each $m, K(m, n)$ decreases and is homogeneous of degree -1 . So, 


$$
\begin{aligned}
\sum_{n=1}^{\infty} K(m, n) & \geqslant \sum_{n=1}^{\infty} \int_{n}^{n+1} K(m, t) d t=\int_{1}^{\infty} K(m, t) d t=m^{-1} \int_{1}^{\infty} K(1, t / m) d t \\
& =m^{-1} \int_{1}^{\infty} K(1, u) m d u=\int_{1}^{\infty} K(1, u) d u
\end{aligned}
$$

and

$$
\begin{aligned}
\sum_{n=-\infty}^{0} K(m, n) & =\sum_{n=0}^{\infty} K(m,-n) \geqslant \sum_{n=1}^{\infty} \int_{n-1}^{n} K(m,-t) d t=m^{-1} \int_{0}^{\infty} K(1,-t / m) d t \\
& =m^{-1} \int_{0}^{-\infty} K(1, u)-m d u=-\int_{0}^{-\infty} K(1, u) d u=\int_{-\infty}^{0} K(1, u) d u .
\end{aligned}
$$

Consequently,

$$
\begin{aligned}
\sum_{n=-\infty}^{\infty} K(m, n) & =\sum_{n=-\infty}^{0} K(m, n)+\sum_{n=1}^{\infty} K(m, n) \\
& \geqslant \int_{-\infty}^{0} K(1, u) d u+\int_{1}^{\infty} K(1, u) d u=\int_{E} K(1, u) d u,
\end{aligned}
$$

where $E=R-[0,1)$.

We, therefore, have

$$
\sum_{n=-\infty}^{\infty}\left[\sum_{m=-\infty}^{\infty} K(m, n) a_{m}\right]^{p} \geqslant A(p, \lambda, K) \sum_{m=-\infty}^{\infty} a_{m}^{p}, \quad p>1 \text { or } p<0 .
$$

In case (ii), we have as in case (i), using inequality (2.9),

$$
\begin{aligned}
\sum_{n=-\infty}^{\infty}\left[\sum_{m=-\infty}^{\infty} K(m, n) a_{m}\right]^{p} & \geqslant \lambda^{p-1} \sum_{n=-\infty}^{\infty} \sum_{m=-\infty}^{n} K(m, n) a_{m}^{p} \\
& =\lambda^{p-1} \sum_{m=-\infty}^{\infty} a_{m}^{p} \sum_{n=m}^{\infty} K(m, n)
\end{aligned}
$$

and the same argument as in case (i) gives us the desired result with $A(p, \lambda, K)=$ $\lambda^{p-1} \int_{1}^{\infty} K(1, u) d u$.

In case (iii), we have as usual,

$\sum_{n=-\infty}^{\infty}\left[\sum_{m=-\infty}^{\infty} K(m, n) a_{m}\right]^{p} \geqslant \lambda^{p-1} \sum_{n=-\infty}^{\infty} \sum_{m=n}^{\infty} K(m, n) a_{m}^{p}=\sum_{m=-\infty}^{\infty} a_{m}^{p} \sum_{n=-\infty}^{m} K(m, n)$.

Now, if $K(m, n)$ increases in $n$, then

$$
\left.\sum_{n=-\infty}^{m} K(m, n) \geqslant \sum_{n=-\infty}^{m} \int_{n-1}^{n} K(m, t) d t=\int_{-\infty}^{m} K(m, t) d t\right) \int_{-\infty}^{1} K(1, u) d u .
$$


If, however, $K(m, n)$ decreases in $n$, we have

$$
\sum_{n=-\infty}^{m} K(m, n) \geqslant \sum_{n=-\infty}^{m} \int_{n}^{n+1} K(m, t) d t=\int_{-\infty}^{m+1} K(m, t) d t \geqslant \int_{-\infty}^{1} K(1, u) d u .
$$

In either case, it follows that inequality (3.1) holds with $A(p, \lambda, K)=\int_{-\infty}^{1} K(1, u) d u$, which proves case (iii) of inequality (3.1).

This completes the proof of inequality (3.1). The proof of inequality (3.2) is similar. Indeed, all we need is to observe that inequalities (3.1) and (3.2) are equivalent. To see this, write $q=1 / p(p>1)$ and $a_{m}^{p}=b_{m}$ in inequality (3.3). We have

$$
\left[\sum_{m=-r}^{s} K(m, n) b_{m}\right]^{q} \geqslant \lambda^{(1 / q)-1} \sum_{m=-r}^{s} K(m, n) b_{m}
$$

i.e.

$$
\left[\sum_{m=-r}^{s} K(m, n) b_{m}\right]^{q} \leqslant \lambda^{q-1} \sum_{m=-r}^{s} K(m, n) b_{m}^{q} .
$$

The same argument as above completes the proof of the theorem.

Numerous interesting applications of the results in this paper provide short methods of obtaining results analogous to those that are similar to Hilbert's double-series theorem (cf. [3, Chapter 9]). Some results in the theory of approximations and on the absolute convergence of Fourier series are further applications of the results of this paper. These applications will appear elsewhere.

\section{REFERENCES}

1. R. P. Boas, Inequalities for monotonic series, J. Math. Anal. Appl. 1 (1960), 121 126. MR 22 \#8244.

2. S. Gatti, Sul massimo di un indice di anormalitá, Metron 18 (1956), no. 1-2, 181 188. MR 18, 683.

3. G. H. Hardy, J. E. Littlewood and G. Pólya, Inequalities, Cambridge Univ. Press, New York, 1934.

4. P. M. Vasić and R. R. Janic', An inequality for convex functions, Univ. Beograd. Publ. Elektrotehn. Fak. Ser. Mat. Fiz. No. 302-319 (1970), 39-42. MR 45 \#8791.

5. J. D. Kěckić and I. B. Lacković, Generalisations of some inequalities of P. M. Vasić, Mat. Vesnik 7 (22) (1970), 74-78. MR 41 \#7050.

6. D. Marković, On an inequality of M. Petrović, Bull. Soc. Math. Phys. Serbie 11 (1959), 45-53. (Serbo-Croatian) MR 32 \#1313.

7. D. S. Mitrinović, Analytic inequalities, Die Grundlehren der math. Wissenschaften, Band 165, Springer-Verlag, New York and Berlin, 1970. MR 43 \#448.

8. - The Steffensen inequality, Univ. Beograd. Publ. Elektrotehn. Fak. Ser. Mat. Fiz. No. 247-273 (1969), 1-14. MR 40 \#5811. 
9. M. Petrović, Sur une fonctionnelle, Publ. Math. Univ. Belgrad 1 (1932), 149-156. 10. J. F. Steffensen, On certain inequalities and methods of approximation, J. Inst. Actuaries 51 (1919), 274-294.

11. P. M. Vasić, Sur une inégalité de M. Petrović, Mat. Vesnik 5 (20) (1968), 473-478. MR 39 \#4343.

P. O. BOX 6413 , WACO, TEXAS 76706

Current address: Department of Mathematics, The University of Ife, Ile-Ife, Western State, Nigeria 